\title{
Ionization cross-sections for positron collisions with $\mathbf{N}_{2}{ }^{\star}$
}

\author{
David A. Cooke ${ }^{\mathrm{a}}$, Daniel J. Murtagh ${ }^{\mathrm{b}}$, and Gaetana Laricchiac \\ UCL Department of Physics and Astronomy, University College London, Gower St, London, WC1E 6BT, UK
}

Received 11 December 2013 / Received in final form 9 January 2014

Published online 25 March 2014

(c) The Author(s) 2014. This article is published with open access at Springerlink.com

\begin{abstract}
The total and direct ionization cross-sections for positron impact on molecular nitrogen have been measured in the energy range from $5 \mathrm{eV}$ to $850 \mathrm{eV}$. The results are compared with other experimental and theoretical determinations.
\end{abstract}

\section{Introduction}

In recent years, a focus in the investigations of positron $\left(\mathrm{e}^{+}\right)$interactions with atoms and molecules has been ionization, a reaction that at low and intermediate energies is often even more significant for positrons than for electrons (e.g. [1,2]). Recent measurements include cross-sections that are differential in energy and/or angle (e.g. $[3,4]$ ), and those relating to ionization accompanied by the excitation of positronium (Ps) [5] and/or of target ion [6]. In the present work, the total and direct ionization crosssections for positron impact on molecular nitrogen at energies in the range $5-850 \mathrm{eV}$ are presented and compared with other experimental and theoretical results.

The main processes by which a nitrogen molecule can be ionized by a low energy positron are annihilation, positronium formation, direct ionization and dissociative ionization (which itself can proceed via direct ionization or positronium formation), as expressed respectively by:

$$
\begin{aligned}
& \mathrm{e}^{+}+\mathrm{N}_{2} \rightarrow \mathrm{N}_{2}^{+}+2 \gamma \\
& \mathrm{e}^{+}+\mathrm{N}_{2} \rightarrow \mathrm{N}_{2}^{+}+\mathrm{Ps}, \\
& \mathrm{e}^{+}+\mathrm{N}_{2} \rightarrow \mathrm{N}_{2}^{+}+\mathrm{e}^{+}+\mathrm{e}^{-}, \\
& \mathrm{e}^{+}+\mathrm{N}_{2} \rightarrow \mathrm{N}^{+}+\mathrm{N}+\mathrm{Ps} /\left(\mathrm{e}^{+}+\mathrm{e}^{-}\right) .
\end{aligned}
$$

Whilst the annihilation channel is open at all energies, the ground-state Ps-formation threshold is given by $E_{P s}=$ $E_{i}-6.8=8.78 \mathrm{eV}$ where $E_{i}$ is the first ionization energy

\footnotetext{
* Contribution to the Topical Issue "Electron and Positron Induced Processes", edited by Michael Brunger, Radu Campeanu, Masamitsu Hoshino, Oddur Ingólfsson, Paulo Limão-Vieira, Nigel Mason, Yasuyuki Nagashima and Hajime Tanuma.

a Present Address: ETH, Institute for Particle Physics, Schafmattstrasse 20, 8093 Zürich, Switzerland

b Present Address: RIKEN, Atomic Physics Laboratory, 351-0198 Saitama, Japan

c e-mail: g.laricchia@ucl.ac.uk
}

of $\mathrm{N}_{2}$ and $6.8 \mathrm{eV}$ the binding energy of ground state Ps. The corresponding total ionization cross-section is usually expressed as:

$$
Q_{i}^{t}=Q_{\mathrm{Ps}}+Q_{i}^{+}+Q_{i}^{\mathrm{diss}}+\sum H O
$$

where $Q_{\mathrm{Ps}}$ is the Ps formation cross-section, $Q_{i}^{+}$the direct single ionization cross-section, $Q_{i}^{\text {diss }}$ the dissociative ionization cross-section, and $\sum H O$ the sum over all higher order processes (such as double and transfer ionization) as well as annihilation, since the latter is considered to give generally a negligible contribution to the total ionization cross-section, as discussed in reference [7].

\section{Experimental apparatus and method}

Details of the present experimental set-up can be found in references $[8,9]$; those elements specific to this work, along with a brief description, are outlined below and illustrated in Figure 1. A beam of approximately $10^{4}$ slow positrons $\mathrm{s}^{-1}$, generated by moderating the fast $\beta^{+}$output of a ${ }^{22} \mathrm{Na}$ source using an annealed $\mathrm{W}$-mesh moderator, is constrained radially by a magnetic field of 100 Gauss. By applying a positive potential $V_{m}$ to the moderator, the peak energy of the beam can be varied according to $E_{+} \approx e V_{m}+2 \mathrm{eV}$. The transmission of unwanted components, for example unmoderated positrons or secondary electrons, is substantially reduced by transporting the beam through a Wien filter and a cylindrical electrode held at $-500 \mathrm{~V}$ prior to the interaction cell. This consists of a hemispherical polished aluminium gas cell from which ions are extracted perpendicularly to the beam axis using a lens held at $-500 \mathrm{~V}$ and are detected using a channel electron multiplier (CEM) with the cone held at $-2800 \mathrm{~V}$. In the present set-up, no $\mathrm{N}^{+}$signal was resolved and, in accordance with an ion optics simulation of the system which indicated a poor efficiency for the extraction of energetic dissociation products, considered negligible. 


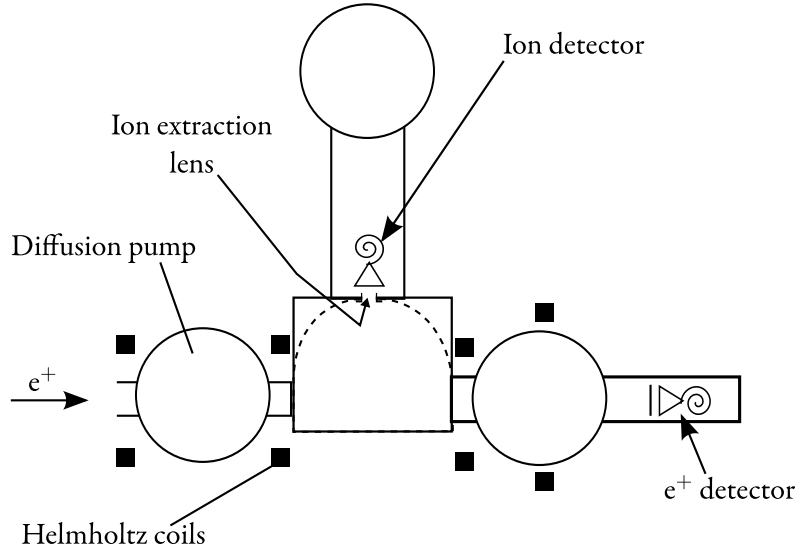

Fig. 1. Interaction region of the beamline showing the positioning of the detectors.

The total yield of ions is defined as:

$$
Y_{i}^{t}(E)=\frac{N_{i}-B_{i}}{N_{+}-B_{+}}
$$

where $N_{i}$ and $N_{+}$are the measured number of ions and positrons respectively, and $B_{i}$ and $B_{+}$represent the associated backgrounds measured by biasing off the slow portion of the positron beam. The absolute scale of the corresponding cross-section is set according to

$$
Q_{i}^{t}(E)=\frac{1}{n l_{\mathrm{eff}}} \frac{\epsilon_{i}^{+} Y_{i}^{t}}{\epsilon_{i}^{i}},
$$

where the $\epsilon_{i}^{+}$and $\epsilon_{i}^{i}$ are the positron- and ion-detector efficiencies and $n l_{\text {eff }}$ is the effective areal gas density, established by the normalization of $Q_{i}^{t}$ for $\operatorname{Ar}$ (determined under identical conditions to the present measurements) to that of reference [10]. The absolute scale thus set yielded values found to agree with $Q_{i}^{t}\left(\mathrm{e}^{-}\right)$at high energies. The latter result is in agreement with the Born approximation according to which the total and direct ionization cross-sections for $\mathrm{e}^{+}$and $\mathrm{e}^{-}$should merge at sufficiently high energies. The direct ionization cross-section $\left(Q_{i}^{+}\right)$is measured simultaneously to $Q_{i}^{t}$ via the corresponding yield $Y_{i}^{+}$, determined by counting coincidences between the ion- and the positron-detectors,

$$
Q_{i}^{+}(E)=\frac{1}{n l_{\mathrm{eff}}} \frac{Y_{i}^{+}}{\epsilon_{i}^{i} \epsilon_{c}}=\frac{Q_{i}^{t}}{Y_{i}^{t}} \frac{Y_{i}^{+}}{\epsilon_{i}^{+} \epsilon_{c} t_{g}^{4}},
$$

where $\epsilon_{c}$ is the efficiency for the coincidence system and $t_{g}$ the transmission coefficient of a grid. The normalization procedure was checked by measuring the equivalent electron cross-section using the same apparatus [6].

\section{Results}

Figure 2 shows the present results for the total ionization cross-section for $\mathrm{N}_{2}^{+}$production compared with the electron data of reference [13] for the non-dissociative

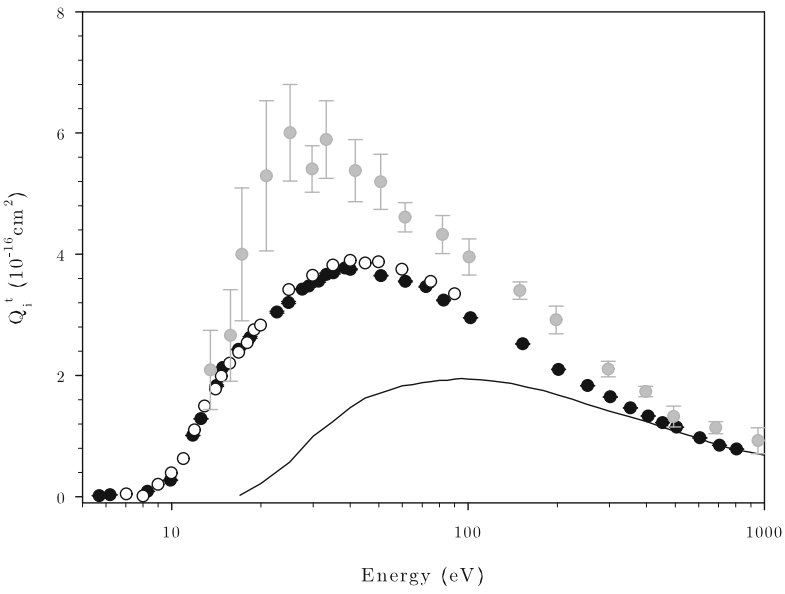

Fig. 2. Measurements of the total non-dissociative ionization cross-section $\left(Q_{i}^{t}\right)$ for positron impact on $\mathrm{N}_{2}$ : present measurements (black circles) and [11] (grey circles), compared with those including dissociative ionization [12] (hollow circles). The corresponding non-dissociative ionization cross-section by electron impact [13] (solid line) is also shown.

component of the cross-section only. The positron crosssection, peaking at $40-50 \mathrm{eV}$ with a magnitude of around $3.8 \times 10^{-16} \mathrm{~cm}^{2}$, exceeds that for electrons, predominantly due to Ps formation, up to approximately $600 \mathrm{eV}$ where they merge. Corresponding determinations of $Q_{i}^{t}$ by [11] display a peak at some $20 \mathrm{eV}$ lower energy with a 50-60\% larger magnitude. Also shown are the results of reference [12] which comprise, unlike the present measurement, possible contributions from dissociative (as well as non-dissociative) ionization. The close correspondence between the two sets below $20 \mathrm{eV}$, and the slight excess of the latter in comparison with the present results above this energy, might imply a crosssection of the order of $0.2 \times 10^{-16} \mathrm{~cm}^{2}$ for $\mathrm{N}^{+}$production (the threshold for which is at $24.29 \mathrm{eV}$ ), a factor of approximately 3.5 lower than than the peak value for electrons [13]. The main dissociative pathway for $\mathrm{N}^{+}$production in single ionization is via the $\mathrm{N}_{2}^{+}\left(C^{2} \Sigma_{u}^{+}\right)$state, the creation of which requires the removal of an innervalence electron [14]. The suppression of the latter type of reactions by a positron has been previously observed (e.g. [6]), although it must be noted that a peak value of approximately $0.8 \times 10^{-16} \mathrm{~cm}^{2}$ has also been reported for positrons by reference [11].

As for $Q_{i}^{t}$, Figure 3 shows the present direct ionization cross-section for $\mathrm{N}_{2}^{+}$production compared with the corresponding electron data of reference [13] which it undercuts both at low and high energies. Whilst the former feature is typical and thought to arise mainly from competition with Ps formation, the latter (amounting to approximately $10 \%$ ) is not and might originate from systematic uncertainties in the normalization [6]. A fair shape agreement may be discerned among all experimental determinations and differences in absolute scale may arise from either the inclusion of dissociation (in the case of Ref. [12]) or normalization methods in comparison with [11]. Indeed, the discrepancy of $\sim 20 \%$ between the present data and 


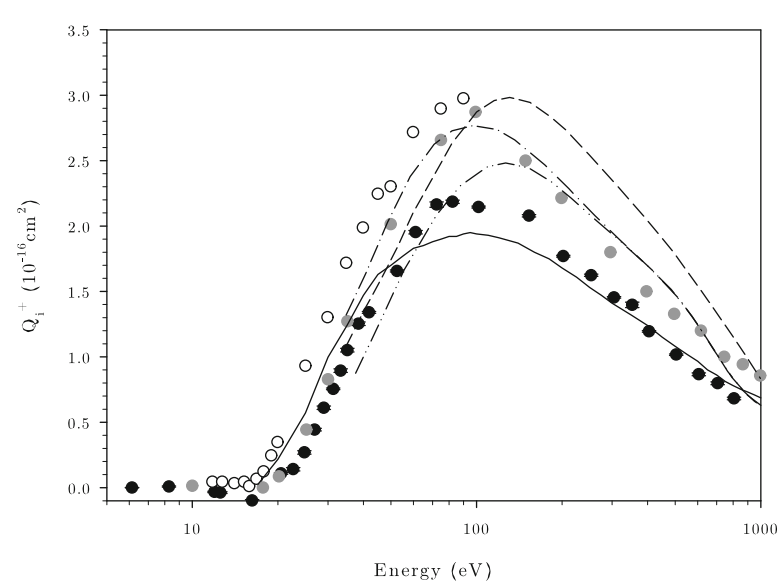

Fig. 3. Measurements of the direct non-dissociative ionization cross-section for positron impact on $\mathrm{N}_{2}$ : present measurements (black circles) and [11] (grey circles), compared with those including dissociative ionization [12] (hollow circles). Also shown are the corresponding non-dissociative ionization cross-section by electron impact [13] (solid line) and the theoretical results of reference [15] corresponding to various models (CPE: short dash; ES: dash dot; TS: dash double dot), as discussed in the text.

that of [11] would be essentially removed if the latter were normalized to the more recent electron data of reference [13] rather than of reference [16]. All three experimental determinations broadly agree on the position of the peak at an energy of $\sim 100 \mathrm{eV}$. Also shown in the figure are the Distorted Wave Born Approximation calculations of reference [15] for the production of $\mathrm{N}_{2}^{+}$ions in which the wavefunctions of the ejected electron and of the incident and scattered positron are calculated in a spherically averaged potential created by the nuclei and the bound electrons (denoted by ES in the figure). Although the present results are generally lower than theory, we note that these theoretical results were improved relative to the CPE model (where the wavefunctions were approximated by Coulomb or plane waves) and total screening (TS) model where the screened potentials have been used for calculating wavefunctions for both the incoming and outgoing particles. The results of the latter two models are also shown in the figure.

\section{Conclusions and outlook}

In conclusion, measurements of $Q_{i}^{t}$ and $Q_{i}^{+}$for $\mathrm{N}_{2}^{+}$production by positrons impact on $\mathrm{N}_{2}$ have been performed from $5 \mathrm{eV}$ to $850 \mathrm{eV}$ and compared with previous determinations for both positrons and electrons. A new gas cell has been designed with improved ion extraction properties which will allow in future the sensitive investigation of dissociative ionization channels.

We are grateful to the Engineering and Physical Sciences Research Council UK for supporting this work under Grant No. EP/E053521/1. We would also like to thank John Dumper and Rafid Jawad for valuable technical support.

\section{References}

1. G. Laricchia, S. Armitage, Á. Kövér, D.J. Murtagh, Adv. At. Mol. Opt. Phys. 56, 1 (2008)

2. G. Laricchia, D.A. Cooke, Á. Kövér, S.J. Brawley, in Experimental Aspects of Ionization Studies by Positron and Positronium Impact (Cambridge University Press, 2013), Vol. 56, pp. 116-136

3. C. Arcidiacono, Á. Kövér, G. Laricchia, Phys. Rev. Lett. 95, $223202(2005)$

4. R.D. DuBois, New J. Phys. 14, 025004 (2012)

5. D.J. Murtagh, D.A. Cooke, G. Laricchia, Phys. Rev. Lett. 102, $133202(2009)$

6. D.A. Cooke, D.J. Murtagh, G. Laricchia, Phys. Rev. Lett. 104, 073201 (2010)

7. P. Van Reeth, G. Laricchia, J.W. Humberston, Phys. Scr. 71, C9 (2005)

8. D.A. Cooke, D.J. Murtagh, Á. Kövér, G. Laricchia, Nucl. Instrum. Methods B 266, 466 (2008)

9. M. Szluinska, P. Van Reeth, G. Laricchia, Nucl. Instrum. Methods B 192, 215 (2002)

10. G. Laricchia, P. Van Reeth, M. Szluinska, J. Moxom, J. Phys. B 35, 2525 (2002)

11. H. Bluhme, N.P. Frandsen, F.M. Jacobsen, H. Knudsen, J.P. Merrison, K. Paludan, M.R. Poulsen, J. Phys. B 31, 4631 (1998)

12. J.P. Marler, C.M. Surko, Phys. Rev. A 72, 062713 (2005)

13. H.C. Straub, P. Renault, B.G. Lindsay, K.A. Smith, R.F. Stebbings, Phys. Rev. A 54, 2146 (1996)

14. O. Dutuit, N. Carrasco, R. Thissen, V. Vuitton, C. Alcaraz, P. Pernot, N. Balucani, P. Casavecchia, A. Canosa, S. Le Picard, J.-C. Loison, Z. Herman, J. Zabka, D. Ascenzi, P. Tosi, P. Franceschi, S.D. Price, P. Lavvas, Astrophys. J. Suppl. Ser. 204, 20 (2013)

15. I. Toth, R.I. Campeanu, V. Chis, L. Nagy, Phys. Lett. A 360, 131 (2006)

16. E. Krishnakumar, S.K. Srivastava, J. Phys. B 23, 1893 (1990)

Open Access This is an open access article distributed under the terms of the Creative Commons Attribution License (http://creativecommons.org/licenses/by/4.0), which permits unrestricted use, distribution, and reproduction in any medium, provided the original work is properly cited. 